Rama, G.; Marinkovic, D.; Zehn, M.

\title{
High performance 3-node shell element for linear and geometrically nonlinear analysis of composite laminates
}

Journal article | Accepted manuscript (Postprint)

This version is available at https://doi.org/10.14279/depositonce-10663

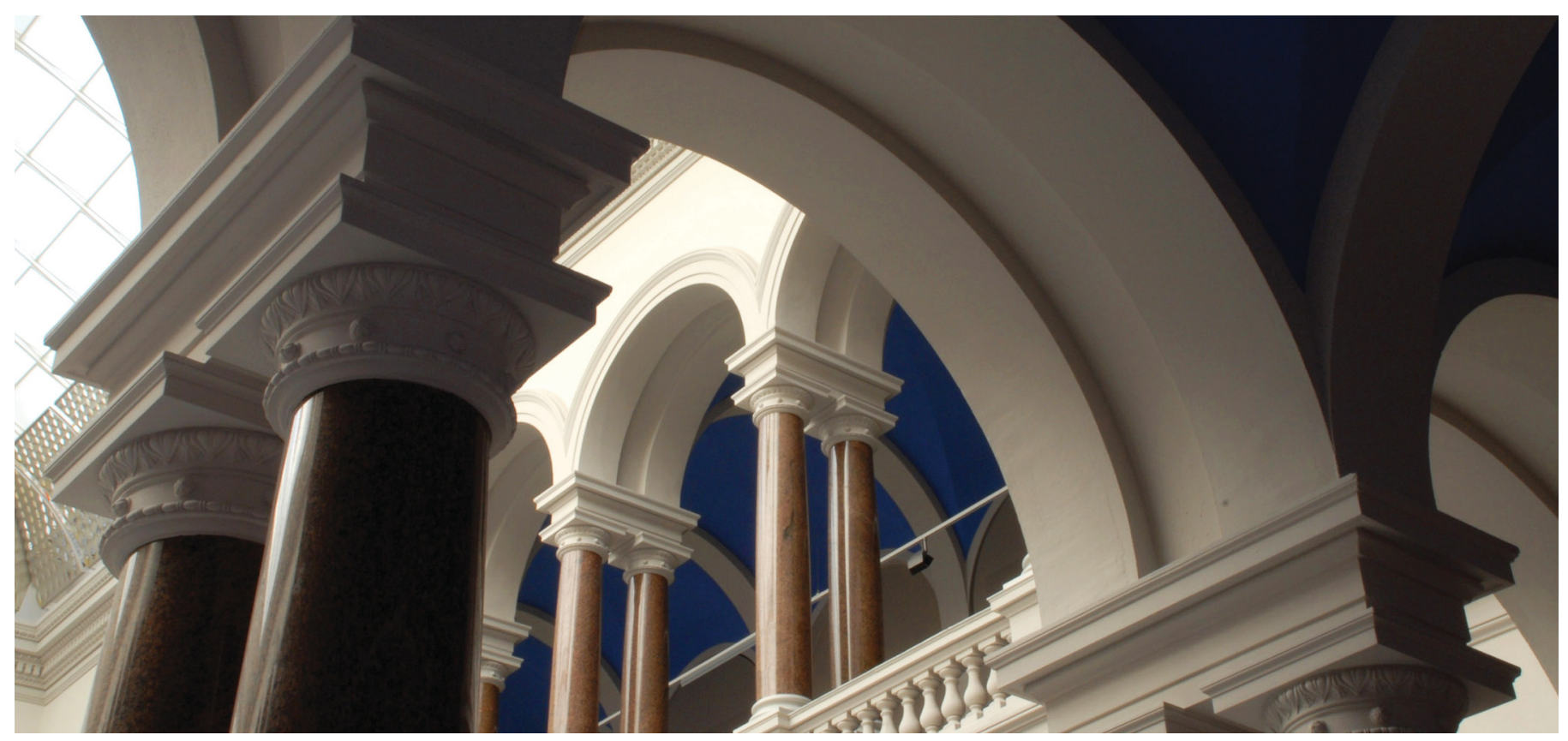

Rama, G., Marinkovic, D., \& Zehn, M. (2018). High performance 3-node shell element for linear and geometrically nonlinear analysis of composite laminates. Composites Part B: Engineering, 151, 118-126. https://doi.org/10.1016/j.compositesb.2018.06.007 


\title{
High performance 3-node shell element for linear and geometrically nonlinear analysis of composite laminates
}

\author{
Gil Rama ${ }^{\mathrm{a}}$, Dragan Marinkovic ${ }^{\mathrm{a}, \mathrm{b}, *}$, Manfred Zehn ${ }^{\mathrm{a}}$ \\ ${ }^{a}$ Department of Structural Analysis, Berlin Institute of Technology, Strasse des 17, Juni \\ 135, 10623 Berlin, Germany \\ ${ }^{b}$ Faculty of Mechanical Engineering, University of Nis, Serbia
}

\begin{abstract}
Thin-walled structures hold primacy among modern engineering structures. All the advantages offered by the curved geometry and thinness of the walls come even more to the fore when combined with exquisite properties of fiber-reinforced composite laminates. Directionally dependant material properties open vast possibilities for tailoring global structural properties and, therewith, optimization. Successful design of such structures calls for high performance shell type finite elements. This paper presents a linear triangular shell element based on the equivalent single-layer approach and the first-order shear deformation theory. The shear locking effect is resolved by the descrete shear gap (DSG) approach combined with the cell smoothing technique. To improve the element performance with respect to the membrane behavior, the assumed natural deviatoric strains (ANDES) formulation is applied, with necessary modifications to meet the requirements of curved structures with anisotropic material properties. Geometric nonlinearities are addressed by the co-rotational formulation. Examples demonstrate the element applicability and performance.
\end{abstract}

Keywords: triangular shell element, discrete shear gap, assumed natural deviatoric strains, fiber-reinforced laminates, co-rotational formulation

\footnotetext{
* Corresponding author

Email address: dragan.marinkovic@tu-berlin.de (Dragan Marinkovic)
} 


\section{Introduction}

With a roughly estimated share of some $80 \%$, thin-walled structures make the group of most commonly encountered engineering structures. This is clearly the consequence of numerous advantages they offer. The thinness of the walls

5 combined with the curved geometry allows the use of high membrane stiffness to carry transversely applied loads. In this manner, a favorable load-to-weight ratio is achieved. The advantages provided by the geometry are further enhanced through application of modern engineering materials, primarily fiber-reinforced composite (FRC) laminates. FRC laminates provide outstanding mechanical 10 properties combined with further weight saving. Also, directionally dependent properties intrinsic for composites make possible tailoring of structural properties already on the material level.

The research on FRC laminated structures is quite diverse, ranging from the work on their general improvements to the work on failure models, detection and localization. As a possibility for further improvement of already exquisite material properties, a number of researchers considered the use of functionally graded materials [1] , 2], [3]. At the same time, a great potential for improvement of general structural properties was seen in application of multi-functional materials, such as piezoelectric materials, which allow active control of their mechanical behavior [4]. Consequently, a great deal of work was dedicated to the development of modeling tools for active composite laminates [5], [6], 7], [8]. On the other side of the research spectrum, due to the proneness of FRC laminates to hidden failures including delamination, research efforts strived to provide reliable models for interlaminar damage and failure of FRC structures,

25 as presented in the survey by Rohwer 9]. Also, methods were developed with the aim of non-destructive damage detection and localization [10], [11]. This rather short glimpse at the research scope related to the composite laminate structures serves only to give a general impression about the attractiveness of the topic.

30 Accurate and reliable modeling and simulation are the prerequisites of success- 
ful research in all the above mentioned research directions. Most frequently the Finite Element Method (FEM), as the method of choice in the field of structural analysis, is used for the purpose. Depending on the research field, models of various complexity and detail levels may be required. This work puts focus onto the global structural behavior. Bearing this in mind, the main workhorse elements in FEM programs are equivalent single-layer shell elements based either on the classical first-order theory (Kirchhoff-Love elements) or the first-order shear deformation theory (Reissner-Mindlin elements). The latter is more general as it includes the consideration of transverse shear effects, which is a rather important aspect for composite laminates. However the main reason why most elements are based on it relies primarily on the reduced continuity requirements from the FE shape functions. The family of degenerate shell elements is a large group of shell elements based on the Reissner-Mindlin kinematics and most of them have also been applied for modeling laminate composite structures [12],

45 [13], 14]. The basic advantage of this element group is the applicability to a relatively wide range of thickness and curvatures. However, they are limited in the aspect of strain and stress recovery in case of laminate structures. Layerwise theories [15], [16] offer a remedy with respect to the stress/strain recovery, but this positive aspect is accompanied by an increased numerical effort. As 50 a well-balanced compromise, Carrera et al. [17] and Valvano and Carrera [18] proposed finite elements with node-dependant kinematics. The approach combines the equivalent single-layer approach and the layer-wise approach. The basic idea is to apply the latter locally in order to provide the adequate accuracy in the structural sub-domains where the strains and stresses are of interest.

${ }_{55}$ Finally, the recently proposed isogeometric approach addresses the problem of seamless integration of design and analysis. The basic idea behind it resides in the use of the same shape functions (NURBS) for both the description of CAD geometry and displacement field of the FE model. Isogeometric developments for composite plates and shells involved the Kirchhoff-Love, Mindlin-Reissner ${ }_{60}$ and higher order kinematics [19], 20], 21], 22].

Obviously, the development of finite elements for shell structures and particu- 
larly those made of composite laminates has attracted a great deal of interest. This paper aims at a high performance triangular shell element based on the first-order shear deformation theory. Geometric nonlinearities are addressed by means of the co-rotational formulation.

\section{Triangular shell element}

The obvious advantages offered by linear triangular elements are the exquisite meshing ability and very high numerical efficiency regarding the computation of matrices and vectors for a single element. As usual, advantages are accompanied by certain disadvantages. Not only may the convergence rate be rather slow due to the ability of the classical linear 3-node element to represent only constant strain and stress states, but the element is also susceptible to the notorious shear locking possibly causing convergence to an erroneous, stiffer solution. To resolve these issues and produce a high performance element, both the bending and membrane behavior of the element will need to be properly modified.

In the element formulation, both the global $(x, y, z)$ and local $\left(x^{\prime}, y^{\prime}, z^{\prime}\right)$ coordinate systems are used, Fig. 1. The local coordinate system is defined so that the $z^{\prime}$-axis is perpendicular to the element surface, while one of the in-plane axes, the $x^{\prime}$-axis, is oriented from element node 1 toward element node 2 . The

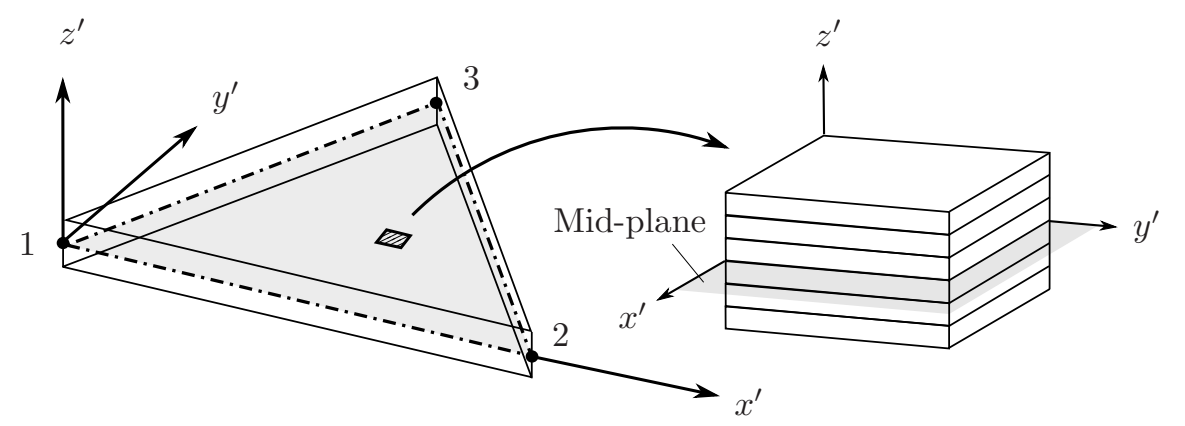

Figure 1: Element geometry, coordinate systems and material architecture 
element employs the classical linear shape functions:

$$
\begin{aligned}
& N_{1}\left(x^{\prime}, y^{\prime}\right)=\frac{1}{2 A}\left(x_{2}^{\prime} y_{3}^{\prime}-x_{3}^{\prime} y_{2}^{\prime}+y_{23}^{\prime} x^{\prime}+x_{32}^{\prime} y^{\prime}\right) \\
& N_{2}\left(x^{\prime}, y^{\prime}\right)=\frac{1}{2 A}\left(x_{3}^{\prime} y_{1}^{\prime}-x_{1}^{\prime} y_{3}^{\prime}+y_{31}^{\prime} x^{\prime}+x_{13}^{\prime} y^{\prime}\right) \\
& N_{3}\left(x^{\prime}, y^{\prime}\right)=\frac{1}{2 A}\left(x_{1}^{\prime} y_{2}^{\prime}-x_{2}^{\prime} y_{1}^{\prime}+y_{12}^{\prime} x^{\prime}+x_{21}^{\prime} y^{\prime}\right)
\end{aligned}
$$

where $x_{i}^{\prime}$ and $y_{i}^{\prime}(i=1,2,3)$, are the local coordinates of the element nodes, $A$ is the element surface area and $x_{i j}^{\prime}$ and $y_{i j}^{\prime}$ denote the abbreviated coordinate differences, i.e. $x_{i j}^{\prime}=x_{i}^{\prime}-x_{j}^{\prime}$ and $y_{i j}^{\prime}=y_{i}^{\prime}-y_{j}^{\prime}$. The element geometry is simply regenerated from its mid-surface:

$$
\left\{\begin{array}{l}
x^{\prime} \\
y^{\prime} \\
z^{\prime}
\end{array}\right\}=\sum_{i=1}^{3} N_{i}\left\{\begin{array}{c}
x_{i}^{\prime} \\
y_{i}^{\prime} \\
0
\end{array}\right\}+\sum_{i=1}^{3} N_{i} \frac{h}{2} \xi\left\{e_{z^{\prime}}\right\}
$$

where $h$ is the element thickness and $\xi$ the natural coordinate $(-1<\xi<+1)$ in the thickness direction. As a consequence of the degeneration process (from 3D to 2D) and the assumed Reissner-Mindlin kinematics, the displacement field $\left\{u^{\prime}, v^{\prime}, w^{\prime}\right\}^{T}$ in the local coordinates is given by:

$$
\left\{\begin{array}{c}
u^{\prime} \\
v^{\prime} \\
w^{\prime}
\end{array}\right\}=\sum_{i=1}^{3} N_{i}\left\{\begin{array}{c}
u_{i}^{\prime} \\
v_{i}^{\prime} \\
w_{i}^{\prime}
\end{array}\right\}+\sum_{i=1}^{3} \frac{h}{2} N_{i} \xi\left\{\begin{array}{c}
\theta_{y^{\prime} i} \\
-\theta_{x^{\prime} i} \\
0
\end{array}\right\}
$$

where $\theta_{x^{\prime}}$ and $\theta_{y^{\prime}}$ are the rotations around the local $x^{\prime}$ - and $y^{\prime}$-axes and $i$ in the right subscript denotes the node number.

Up to this point, all the equations fit into the classical formulation. However, as already discussed above, the strain field directly derived using the kinematic relations produces a too stiff element that suffers sub-optimal convergence or even a convergence toward an erroneous solution. Hence, special techniques are needed as a remedy. Since a flat element is considered here, its deformational behavior can be represented as a superposition of plate and membrane elements.

75 The development presented here implements already existing solutions for both bending and membrane behavior, but it represents a novel combination of those 
solutions. In what follows, the basic ideas and most important formulae are given as the available literature that is referenced below provides the necessary details.

\subsection{Plate behavior}

Since the element is based on the first-order shear deformation theory, the stiffness matrix of the plate element consists of the bending stiffness and transverse shear stiffness. Using the discretized displacement field (Eq. (3)), the bending strains with respect to the local coordinate system are directly derived:

$$
\left\{\begin{array}{c}
\varepsilon_{x^{\prime} x^{\prime}}^{b} \\
\varepsilon_{y^{\prime} y^{\prime}}^{b} \\
\gamma_{x^{\prime} y^{\prime}}^{b}
\end{array}\right\}=z^{\prime}\left\{\begin{array}{c}
\frac{\partial \theta_{y^{\prime}}}{\partial x^{\prime}} \\
-\frac{\partial \theta_{x^{\prime}}}{\partial y^{\prime}} \\
\frac{\partial \theta_{y^{\prime}}}{\partial y^{\prime}}-\frac{\partial \theta_{x^{\prime}}}{\partial x^{\prime}}
\end{array}\right\}=z^{\prime} \sum_{i=1}^{3}\left[\begin{array}{ccc}
0 & 0 & N_{i, x^{\prime}} \\
0 & -N_{i, y^{\prime}} & 0 \\
0 & -N_{i, x^{\prime}} & N_{i, y^{\prime}}
\end{array}\right]\left\{\begin{array}{c}
w_{i}^{\prime} \\
\theta_{x^{\prime} i} \\
\theta_{y^{\prime} i}
\end{array}\right\}
$$

thus yielding the corresponding strain-displacement matrix $\left[B_{p b}\right]$ in the straightforward form:

$$
\left[B_{p b}\right]=\frac{1}{2 A}\left[\begin{array}{ccccccccc}
0 & 0 & y_{23}^{\prime} & 0 & 0 & y_{31}^{\prime} & 0 & 0 & y_{12}^{\prime} \\
0 & x_{32}^{\prime} & 0 & 0 & x_{13}^{\prime} & 0 & 0 & x_{21}^{\prime} & 0 \\
y_{23}^{\prime} & x_{32}^{\prime} & 0 & y_{31}^{\prime} & x_{13}^{\prime} & 0 & y_{12}^{\prime} & x_{21}^{\prime} & 0
\end{array}\right]
$$

Due to the susceptibility of the element to shear locking, a more sophisticated approach is required. Exactly the same technique as the one used in the formulation of the piezoelectric 3-node element by Marinkovic and Rama 23] is applied here as well. It is the descrete shear gap (DSG) technique originally proposed by Bletzinger et al. [24], the basic idea of which is in the separation of the deformation into the part due to transverse shear and a part due to bending. This further allows extraction of the part that is only due to transverse shear, denoted as shear gap. The shear gaps are then evaluated at the nodes, interpolated using the linear shape functions given in Eq. (11) and finally differentiated to obtain the transverse shear field based on the DSG approach. When used in combination with a linear triangular plate element, the resulting strain-displacement matrix with respect to the local coordinate system, $\left[B_{s}\right]$, 
has the following closed form:

$$
\left[B_{s}\right]=\frac{1}{2 A}\left[\begin{array}{ccccccccc}
x_{32}^{\prime} & A & 0 & x_{13}^{\prime} & a_{1} & a_{3} & x_{21}^{\prime} & -a_{2} & -a_{3} \\
y_{23}^{\prime} & 0 & -A & y_{31}^{\prime} & a_{4} & a_{2} & y_{12}^{\prime} & -a_{4} & -a_{1}
\end{array}\right]
$$

with the following geometric parameters:

$$
\begin{array}{ll}
a_{1}=\frac{1}{2} y_{12}^{\prime} x_{13}^{\prime} & a_{2}=\frac{1}{2} y_{31}^{\prime} x_{21}^{\prime} \\
a_{3}=\frac{1}{2} x_{21}^{\prime} x_{13}^{\prime} & a_{4}=\frac{1}{2} y_{12}^{\prime} y_{31}^{\prime}
\end{array}
$$

This approach tackles the shear locking effects, but notable shear stress oscillations between elements may still appear. The so-called cell-smoothing technique 25] is applied as a remedy. The idea is relatively simple. The element is divided into three sub-elements using the element centroid as an additional node. Equation (6) is applied to each of the sub-elements. Additionally, the displacement at the element centroid is interpolated from the nodal displacements, i.e. it is their mean value. This results in three strain-displacements matrices, $\left[B_{s}^{\Delta_{j}}\right]$, $j=1,2,3$, each one for one of the sub-elements:

$$
\begin{aligned}
& {\left[B_{s}^{\Delta_{1}}\right]=\left[\left[\frac{1}{3} B_{s 1}^{\Delta_{1}}+B_{s 3}^{\Delta_{1}}\right]\left[\frac{1}{3} B_{s 1}^{\Delta_{1}}\right]\left[\frac{1}{3} B_{s 1}^{\Delta_{1}}+B_{s 2}^{\Delta_{1}}\right]\right]} \\
& {\left[B_{s}^{\Delta_{3}}\right]=\left[\left[\frac{1}{3} B_{s 1}^{\Delta_{3}}+B_{s 2}^{\Delta_{3}}\right]\left[\frac{1}{3} B_{s 1}^{\Delta_{3}}+B_{s 3}^{\Delta_{3}}\right]\left[\frac{1}{3} B_{s 1}^{\Delta_{3}}\right]\right]} \\
& {\left[B_{s}^{\Delta_{2}}\right]=\left[\left[\frac{1}{3} B_{s 1}^{\Delta_{2}}\right]\left[\frac{1}{3} B_{s 1}^{\Delta_{2}}+B_{s 2}^{\Delta_{2}}\right]\left[\frac{1}{3} B_{s 1}^{\Delta_{2}}+B_{s 3}^{\Delta_{3}}\right]\right]}
\end{aligned}
$$

where $\left[B_{s i}^{\Delta_{j}}\right](i=1,2,3)$, is the transverse shear strain-displacement matrix of the $i^{\text {th }}$ element node. Finally, the strain-displacement matrix related to the transverse shear is obtained as a mean value of the matrices given in Eq. (8):

$$
\left[B_{p s}\right]=\frac{1}{3} \sum_{i=1}^{3}\left[B_{s}^{\triangle_{i}}\right]
$$

Using the well-known ABD material matrix for composite laminates 26], the stiffness matrix for the plate part of the element is given as:

$$
\left[K_{p}\right]=\int_{(A)}\left[B_{p b}\right]^{T}[D]\left[B_{p b}\right] \mathrm{d} A+\int_{(A)}\left[B_{p s}\right]^{T}[F]\left[B_{p s}\right] \mathrm{d} A
$$

where $[F]$ is the transverse shear stiffness of the composite laminate computed according to Rolfes and Rohwer [27]. 


\subsection{Membrane behavior}

The membrane part of the element is resolved using the assumed natural deviatoric strain (ANDES) formulation by Felippa and Militello [28]. The idea behind this approach is representation of the displacement field by a carefully selected set of modes. Hence, the discretized displacement field given by Eq. (3) is abandoned for the membrane part. The selected set of mode shapes is a complete set of linearly independent modes comprising basic modes, i.e. rigid-body and constant strain modes, and higher order modes. In the case of the linear triangular element, the higher order modes are the linear strain modes. These are actually the modes that cannot be represented by the classical formulation of the element and, hence, this is where the additional quality is introduced. The overall number of linearly independent modes has to be the same as the number of degrees of freedom, which also guarantees the rank sufficiency of the element stiffness matrix. If the selected modes are not linearly independent, then a sufficient number of additional kinematic constraints are to be introduced. Finally, the variational correctness is kept by enforcing the orthogonality of the linear strain modes and the constant strain modes.

Analogously to the grouping of the modes into basic and higher order modes, the membrane stiffness also comprises the basic, $\left[K_{b}\right]$, and higher order stiffness, $\left[K_{h}\right]:$

$$
\left[K_{m e m}\right]=\left[K_{b}\right]+\left[K_{h}\right]
$$

The basic stiffness is determined in the same manner as in the free formulation by Bergan and Nygard [29], but modified to account for the composite laminate material architecture:

$$
\left[K_{b}\right]=(V h)^{-1}[L][A][L]^{T}
$$

where $V$ is the element volume, $[A]$ is the laminate membrane stiffness contained in the $\mathrm{ABD}$ matrix, while $[L]$ is the force-lumping matrix that consistently maps 
an arbitrary constant stress field into the element nodal forces [29]:

$$
[L]=\frac{h}{2}\left[\begin{array}{ccc}
y_{23}^{\prime} & 0 & x_{32}^{\prime} \\
0 & x_{32}^{\prime} & y_{23}^{\prime} \\
\frac{\alpha}{6} y_{23}^{\prime}\left(y_{32}^{\prime}-y_{12}^{\prime}\right) & \frac{\alpha}{6} x_{32}^{\prime}\left(x_{31}^{\prime}-x_{12}^{\prime}\right) & \frac{\alpha}{3}\left(x_{31}^{\prime} y_{13}^{\prime}-x_{12}^{\prime} y_{21}^{\prime}\right) \\
y_{31}^{\prime} & 0 & x_{13}^{\prime} \\
0 & x_{13}^{\prime} & y_{31}^{\prime} \\
\frac{\alpha}{6} y_{31}^{\prime}\left(y_{32}^{\prime}-y_{12}^{\prime}\right) & \frac{\alpha}{6} x_{13}^{\prime}\left(x_{12}^{\prime}-x_{23}^{\prime}\right) & \frac{\alpha}{3}\left(x_{12}^{\prime} y_{21}^{\prime}-x_{23}^{\prime} y_{32}^{\prime}\right) \\
y_{12}^{\prime} & 0 & x_{21}^{\prime} \\
0 & x_{21}^{\prime} & y_{12}^{\prime} \\
\frac{\alpha}{6} y_{12}^{\prime}\left(y_{32}^{\prime}-y_{12}^{\prime}\right) & \frac{\alpha}{6} x_{21}^{\prime}\left(x_{23}^{\prime}-x_{31}^{\prime}\right) & \frac{\alpha}{3}\left(x_{23}^{\prime} y_{32}^{\prime}-x_{31}^{\prime} y_{13}^{\prime}\right)
\end{array}\right]
$$

with $\alpha$ denoting a free parameter that will be discussed later.

The higher order stiffness allows the element to properly recover the in-plane bending. For this purpose, three in-plane bending modes that induce linear strains within the element are used. The drilling degree of freedom (rotation about the normal), $\theta_{z^{\prime}}$, plays the major role in the description of these modes. Without going into all the details, which are available in [30], 31], it is important to emphasize that the modes are selected so that the drilling degree of freedom at one of the nodes is set to zero, while the values at the remaining two nodes have the same absolute value, but are of the opposite sign. Also, the element is (in-plane) bent so that the center of curvature is on the side of the node with the zero drilling degree of freedom. Since the so selected three modes are not linearly independent, an additional, (in-plane) torsional mode is introduced, in which the drilling degree of freedom at each node has the same value. This approach leads to the following form of the higher order membrane stiffness:

$$
\left[K_{h}\right]=\beta_{0} \frac{9}{4}\left[T_{\theta u}\right]^{T}\left[K_{\theta}\right]\left[T_{\theta u}\right]
$$

where $\beta_{0}$ is one of the dimensionless parameters and:

$$
\left[T_{\theta u}\right]=\frac{1}{4 A}\left[\begin{array}{ccccccccc}
x_{32}^{\prime} & y_{32}^{\prime} & 4 A & x_{13}^{\prime} & y_{13}^{\prime} & 0 & x_{21}^{\prime} & y_{21}^{\prime} & 0 \\
x_{32}^{\prime} & y_{32}^{\prime} & 0 & x_{13}^{\prime} & y_{13}^{\prime} & 4 A & x_{21}^{\prime} & y_{21}^{\prime} & 0 \\
x_{32}^{\prime} & y_{32}^{\prime} & 0 & x_{13}^{\prime} & y_{13}^{\prime} & 0 & x_{21}^{\prime} & y_{21}^{\prime} & 4 A
\end{array}\right]
$$




$$
\left[K_{\theta}\right]=\frac{1}{3}\left(\left[Q_{4}\right]^{T}\left[A_{n a t}\right]\left[Q_{4}\right]+\left[Q_{5}\right]^{T}\left[A_{n a t}\right]\left[Q_{5}\right]+\left[Q_{6}\right]^{T}\left[A_{n a t}\right]\left[Q_{6}\right]\right)
$$

where $\left[A_{\text {nat }}\right]$ is given by:

$$
\left[A_{n a t}\right]=\left[T_{n a t}\right]^{T}[A]\left[T_{n a t}\right]
$$

with the following transformation matrix between the so-called natural orientations and the Cartesian orientations:

$$
\left[T_{n a t}\right]=\frac{1}{4 A^{2}}\left[\begin{array}{lll}
y_{23}^{\prime} y_{13}^{\prime} l_{21}^{2} & x_{23}^{\prime} x_{13}^{\prime} l_{21}^{2} & \left(y_{23}^{\prime} x_{31}^{\prime}+x_{32}^{\prime} y_{13}^{\prime}\right) l_{21}^{2} \\
y_{31}^{\prime} y_{21}^{\prime} l_{32}^{2} & x_{31}^{\prime} x_{21}^{\prime} l_{32}^{2} & \left(y_{31}^{\prime} x_{12}^{\prime}+x_{13}^{\prime} y_{21}^{\prime}\right) l_{32}^{2} \\
y_{12}^{\prime} y_{32}^{\prime} l_{13}^{2} & x_{12}^{\prime} x_{32}^{\prime} l_{13}^{2} & \left(y_{12}^{\prime} x_{23}^{\prime}+x_{21}^{\prime} y_{32}^{\prime}\right) l_{13}^{2}
\end{array}\right]^{T}
$$

The $Q$-matrices in Eq. (16) are obtained in the following manner:

$$
\begin{aligned}
& {\left[Q_{4}\right]=\frac{1}{2}\left(\left[Q_{1}\right]+\left[Q_{2}\right]\right)} \\
& {\left[Q_{5}\right]=\frac{1}{2}\left(\left[Q_{2}\right]+\left[Q_{3}\right]\right)} \\
& {\left[Q_{6}\right]=\frac{1}{2}\left(\left[Q_{1}\right]+\left[Q_{3}\right]\right)}
\end{aligned}
$$

with

$$
\begin{gathered}
{\left[Q_{1}\right]=\frac{2 A}{3}\left[\begin{array}{lll}
\frac{\beta_{1}}{l_{21}^{2}} & \frac{\beta_{2}}{l_{21}^{2}} & \frac{\beta_{3}}{l_{21}^{2}} \\
\frac{\beta_{4}}{l_{32}^{2}} & \frac{\beta_{5}}{l_{32}^{2}} & \frac{\beta_{6}}{l_{32}^{2}} \\
\frac{\beta_{7}}{l_{13}^{2}} & \frac{\beta_{8}}{l_{13}^{2}} & \frac{\beta_{9}}{l_{13}^{2}}
\end{array}\right]} \\
{\left[Q_{2}\right]=\frac{2 A}{3}\left[\begin{array}{lll}
\frac{\beta_{9}}{l_{21}^{2}} & \frac{\beta_{7}}{l_{21}^{2}} & \frac{\beta_{8}}{l_{21}^{2}} \\
\frac{\beta_{3}}{l_{32}^{2}} & \frac{\beta_{1}}{l_{32}^{2}} & \frac{\beta_{2}}{l_{32}^{2}} \\
\frac{\beta_{6}}{l_{13}^{2}} & \frac{\beta_{4}}{l_{13}^{2}} & \frac{\beta_{5}}{l_{13}^{2}}
\end{array}\right]} \\
{\left[Q_{3}\right]=\frac{2 A}{3}\left[\begin{array}{lll}
\frac{\beta_{5}}{l_{21}^{2}} & \frac{\beta_{6}}{l_{21}^{2}} & \frac{\beta_{4}}{l_{21}^{2}} \\
\frac{\beta_{8}}{l_{32}^{2}} & \frac{\beta_{9}}{l_{32}^{2}} & \frac{\beta_{7}}{l_{32}} \\
\frac{\beta_{2}}{l_{13}^{2}} & \frac{\beta_{3}}{l_{13}^{2}} & \frac{\beta_{1}}{l_{13}^{2}}
\end{array}\right]}
\end{gathered}
$$

The choice of free parameters $\alpha$ and $\beta_{0}-\beta_{9}$ aims at optimal membrane behavior of a shell element for composite laminates. Fellipa [30] provided the optimal values of $\alpha$ and $\beta$-parameters for the purely membrane triangular element. On the other hand, the deformational behavior that involves both membrane and 
bending deformation and their coupling requires modified values. Shin and Lee 32] demonstrated that the following values represent a good choice for the shell

90 element: $\alpha=\frac{1}{8}, \beta_{0}=\alpha^{2} / 4, \beta_{1}=1, \beta_{2}=2, \beta_{3}=1, \beta_{4}=0, \beta_{5}=1, \beta_{6}=-1$, $\beta_{7}=-1, \beta_{8}=-1, \beta_{9}=-2$.

\section{Co-rotational formulation}

In the previous two decades, the co-rotational (CR) approach attracted significant attention as a $\mathrm{FE}$ formulation suitable for structural deformations characterized by large rotations but small strains [33], 34], [35], [36]. Geometrically nonlinear deformations intrinsic for thin-walled structures in the working regimes mostly fit into this description.

The key idea behind the CR approach is to decompose the overall motion into the rigid-body motion and pure deformation. This is achieved by introducing a co-rotational coordinate system (CR frame) that is attached to the element and performs the same rigid-body motion as the element, Fig. 2. The pure deformations are then measured in the CR frame. The assumption of small
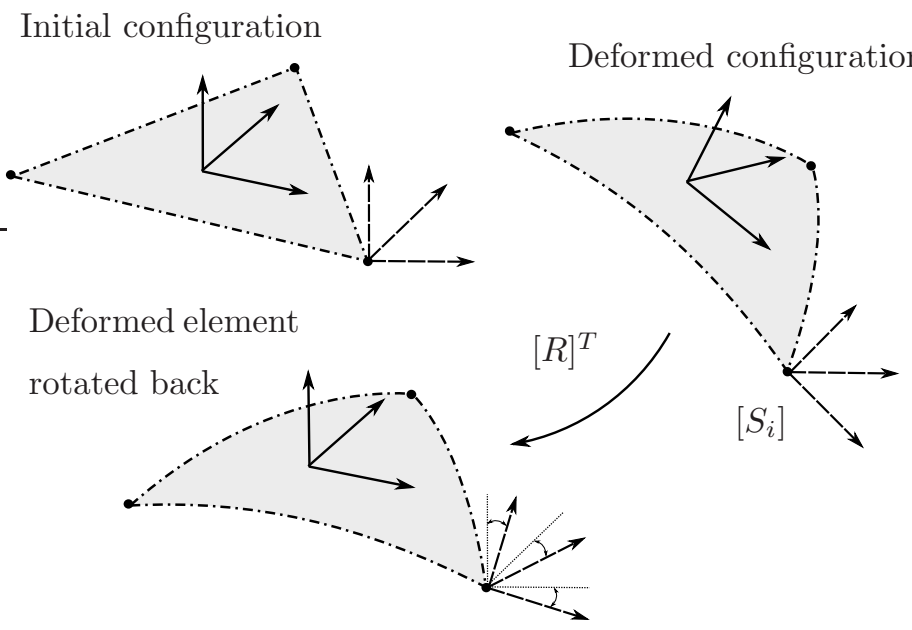

Deformed configuration

Figure 2: Co-rotational approach

strains allows the use of the matrices and vectors developed in the linear element formulation, which counts for one of the main advantages of the approach. 
The present element is extended to cover the geometrically nonlinear analysis, whereby the CR formulation by Nour-Omid and Rankin [37] is adopted. The following relations are used to obtain the deformational nodal translations and rotations:

$$
\begin{gathered}
\left\{\bar{u}_{i}^{e}\right\}=[E]^{T}\left(\left\{u_{i}^{g}\right\}+\left\{X_{i}^{g}\right\}-\left\{u_{0}^{g}\right\}\right)-\left\{X_{i}^{e}\right\} \\
{\left[\bar{T}_{i}\right]=[E]^{T}\left[S_{i}\right]\left[E_{0}\right]}
\end{gathered}
$$

where the subscripting $i$ denotes the node number, the superscripting $g$ and $e$ mean that the quantity is given in the global and CR frames, respectively, $\left\{\bar{u}_{i}\right\}$ are the deformational translations, $\left\{u_{i}\right\}$ are the global translations, $\left\{u_{0}\right\}$ the translation of the $\mathrm{CR}$ frame origin, and $\left[\bar{T}_{i}\right]$ is the rotational matrix associated with the deformational rotations. $[E]$ and $\left[E_{0}\right]$ are the element $\mathrm{CR}$ frames in the current and initial configurations, respectively, $\{X\}$ is the initial element configuration (i.e. nodal coordinates), and finally, $\left[S_{i}\right]$ is the total rotational matrix of the nodal triad $[38]$ :

$$
\left[S_{i}\right]=[I]+\frac{\sin \left(\gamma_{i}\right)}{\gamma_{i}}\left[N_{i}\right]+\frac{1}{2}\left(\frac{\sin \left(\gamma_{i} / 2\right)}{\left(\gamma_{i} / 2\right)}\right)^{2}\left[N_{i}\right]^{2}
$$

where:

$$
\gamma_{i}=\sqrt{\theta_{x i}^{2}+\theta_{y i}^{2}+\theta_{z i}^{2}}
$$

and:

$$
\left[N_{i}\right]=\left[\begin{array}{ccc}
0 & -\theta_{z i} & \theta_{y i} \\
\theta_{z i} & 0 & -\theta_{x i} \\
-\theta_{y i} & \theta_{x i} & 0
\end{array}\right]
$$

After several transformations, the variation of Eqs. (21) and (22) can be represented in the following form [37]:

$$
\begin{gathered}
\delta\left\{\bar{u}_{i}^{e}\right\}=\operatorname{Spin}\left(\left\{x_{i}^{e}\right\}\right) \delta\left\{\omega_{E}^{e}\right\}+\delta\left\{u_{i}^{e}\right\} \\
\delta\left[\bar{T}_{i}\right]=\left(\operatorname{Spin}\left(\delta\left\{\omega_{S}^{e}\right\}\right)-\operatorname{Spin}\left(\delta\left\{\omega_{E}^{e}\right\}\right)\right)\left[\bar{T}_{i}\right]
\end{gathered}
$$


where $\delta\left\{\omega_{E}^{e}\right\}$ and $\delta\left\{\omega_{S}^{e}\right\}$ are the variations (spin) of triads $[E]$ and $\left[S_{i}\right]$ in the element $(\mathrm{CR})$ coordinates, $\left\{x_{i}^{e}\right\}$ are the current coordinates of the $i^{\text {th }}$ node in the CR frame, while Spin denotes a skew-symmetric spin tensor built from a 3-dimensional rotation vector $\{\omega\}=\left\{\omega_{1}, \omega_{2}, \omega_{3}\right\}^{T}$ :

$$
\operatorname{Spin}(\{\omega\})=\left[\begin{array}{ccc}
0 & -\omega_{3} & \omega_{2} \\
\omega_{3} & 0 & -\omega_{1} \\
-\omega_{2} & \omega_{1} & 0
\end{array}\right]
$$

The fact that the internal forces can be defined in two ways - as a derivative of the strain energy with respect to the global displacements, but also as a derivative of the strain energy with respect to the deformational displacements leads to a relation between the internal forces in the global and in the local coordinate systems by means of the generalized Jacobian transformation matrix between the global displacements and their deformational counterpart in the CR frame. This matrix, $[P]$, has a property of a projector matrix and is given as [37]:

$$
[P]=\left[\begin{array}{ll}
\partial\{\bar{u}\}^{T} / \partial\{u\} & \partial\{\bar{\theta}\}^{T} / \partial\{u\} \\
\partial\{\bar{u}\}^{T} / \partial\{\theta\} & \partial\{\bar{\theta}\}^{T} / \partial\{\theta\}
\end{array}\right]=[I]-[\Psi][\Gamma]^{T}
$$

where $[I]$ is the identity matrix, $[\Psi]$ reads $[37]$ :

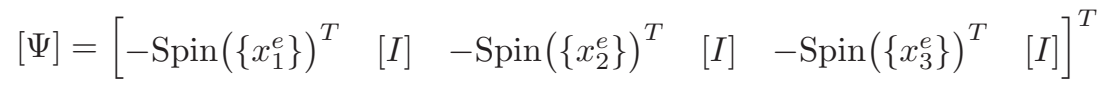

and the nodal $\left[\Gamma_{i}\right][37]$ :

$$
\left[\Gamma_{i}\right]=\left[\begin{array}{l}
\partial\left\{\omega_{E}\right\}^{T} / \partial\left\{u_{i}\right\} \\
\partial\left\{\omega_{E}\right\}^{T} / \partial\left\{\theta_{i}\right\}
\end{array}\right]
$$

In Eq. (31) the upper part of the matrix is associated with translations, while the lower part with rotations. In case of the triangular element, the $[\Gamma]$ matrix is computed as:

$$
\left.[\Gamma]=\left[\begin{array}{llll}
{\left[\Gamma_{1}\right]^{T}} & {[0]} & {\left[\Gamma_{2}\right.}
\end{array}\right]^{T} \quad[0] \quad\left[\Gamma_{3}\right]^{T} \quad[0]\right]^{T}
$$


with:

$$
\begin{aligned}
{\left[\Gamma_{1}\right]^{T} } & =\left[\begin{array}{ccc}
0 & 0 & -1 / y_{3}^{e} \\
0 & 0 & \left(1 / x_{2}^{e}\right)\left(1-y_{2}^{e}\right) / y_{3}^{e} \\
1 / y_{3}^{e} & 0 & 0
\end{array}\right] \\
{\left[\Gamma_{2}\right]^{T} } & =\left[\begin{array}{ccc}
0 & 0 & 0 \\
0 & 0 & -1 / x_{2}^{e} \\
0 & 0 & 0
\end{array}\right] \\
{\left[\Gamma_{3}\right]^{T} } & =\left[\begin{array}{ccc}
0 & 0 & 1 / y_{3}^{e} \\
0 & 0 & y_{2}^{e} /\left(x_{2}^{e} y_{3}^{e}\right) \\
-1 / y_{3}^{e} & 0 & 0
\end{array}\right]
\end{aligned}
$$

where $x_{i}^{e}, y_{i}^{e}$ are the first two components of $\left\{x_{i}^{e}\right\}$. Finally, with the projector matrix defined, the stiffness matrix $\left[K^{g}\right]$ and internal forces $\left\{f_{\text {int }}^{g}\right\}$ in the global coordinate system are computed as follows:

$$
\begin{gathered}
{\left[K^{g}\right]=[E][P]^{T}\left[K^{e}\right][P][E]^{T}} \\
\left\{f_{\text {int }}^{g}\right\}=[E][P]^{T}\left[K^{e}\right]\left\{\left\{\bar{u}^{e}\right\}^{T}\left\{\bar{\theta}^{e}\right\}^{T}\right\}^{T}
\end{gathered}
$$

\section{Numerical examples}

In order to validate the developed element and examine its properties, several

95 practical examples, linear and geometrically nonlinear, and the verification is done by comparing the obtained results with those yielded by the shell elements of other authors or the S3 element from Abaqus, while for the practical example also the experimental results are used. Some of the considered examples are proposed by various authors and involve different systems of units. To avoid any inconsistency in the units used, all numerical values are given as dimensionless. As a matter of fact, in each example, any set of units can be associated with the values as long as it is used consistently throughout the example. 


\subsection{Laminated strip under three-point bending}

105 NAFEMS [39] as a linear static composite benchmark case [40]. The laminate has a stacking sequence $[0 / 90 / 0 / 90 / 0 / 90 / 0]$ with respect to the material reference direction, which is the global $x$-axis. The layer thicknesses are given in Fig. 3. while Table 1 provides the material properties. The laminated strip is subjected to a constant line load of $q=10$ acting along the mid-span of the structure as shown in Fig. 1, above. The symmetry of the structure and ap-
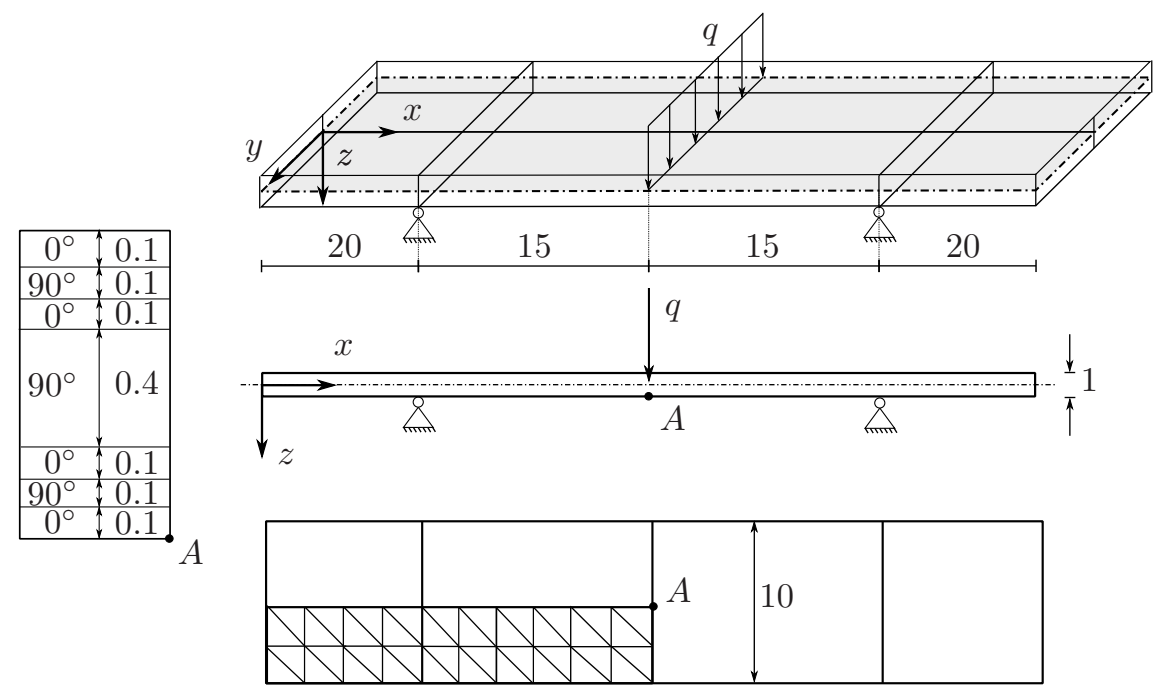

Figure 3: Laminated strip under three-point bending

Table 1: Material properties - laminated strip

\begin{tabular}{cccccc}
\hline$E_{1}$ & $E_{2}$ & $\nu_{12}$ & $G_{12}$ & $G_{13}$ & $G_{23}$ \\
\hline $100 \cdot 10^{9}$ & $5 \cdot 10^{9}$ & 0.4 & $3 \cdot 10^{9}$ & $2 \cdot 10^{9}$ & $2 \cdot 10^{9}$ \\
\hline
\end{tabular}

plied boundary conditions allow modeling of only one quarter of the structure with an appropriate extension of the kinematic boundary conditions (symmetry along the internal edges). The vertical displacement and the in-plane bending 115 
for several meshes using the present element and its Abaqus counterpart. As it is generally known, the results for displacements converge typically faster than those for stresses. This is also the case here and both elements yield the converged result for the displacement already with the roughest mesh used. For that reason, only the convergence for the stress result is given in Table 2 which obviously shows rather similar convergence rate of both elements. The results in Table 2 are normalized by the reference solution reported in the NAFEMS publication [40].

Table 2: Convergence data - bending stress at point A normailized by 684 [40]

\begin{tabular}{ccc}
\hline Elements & Present & Abaqus S3 \\
\hline 14 & 0.884 & 0.879 \\
32 & 0.904 & 0.908 \\
120 & 0.952 & 0.952 \\
528 & 0.980 & 0.982 \\
800 & 0.989 & 0.988 \\
\hline
\end{tabular}

\subsection{Clamped angle-ply cylindrical composite panel}

The case of the clamped angle-ply cylindrical composite panel depicted in Fig. 4 was proposed by Yeom and Lee [4] and later extended by Kulikov and Plotnikova 42]. The focus in the example is on the geometrically nonlinear behavior of the anisotropic shell structure. The curved edges are free, while the straight edges are clamped. The geometry and dimensions are shown in Fig. 4 The total shell thickness is $h=0.496$ and it includes two plies of equal thickness, that is 0.248 . The $\alpha_{1}$-direction shown in Fig. 4 is the material reference direction. Whereas Yeom and Lee [1]] considered only the ply-laminate with the ply sequence [45/-45], Kulikov and Plotnikova [42] extended the example by another two stacking sequences: [75/-75] and [15/-15]. The material properties are given in Table 3. The shell structure is exposed to a concentrated force $f=10000$ acting at the center point, Fig. 4. The results reported by Yeom and Lee [41] 


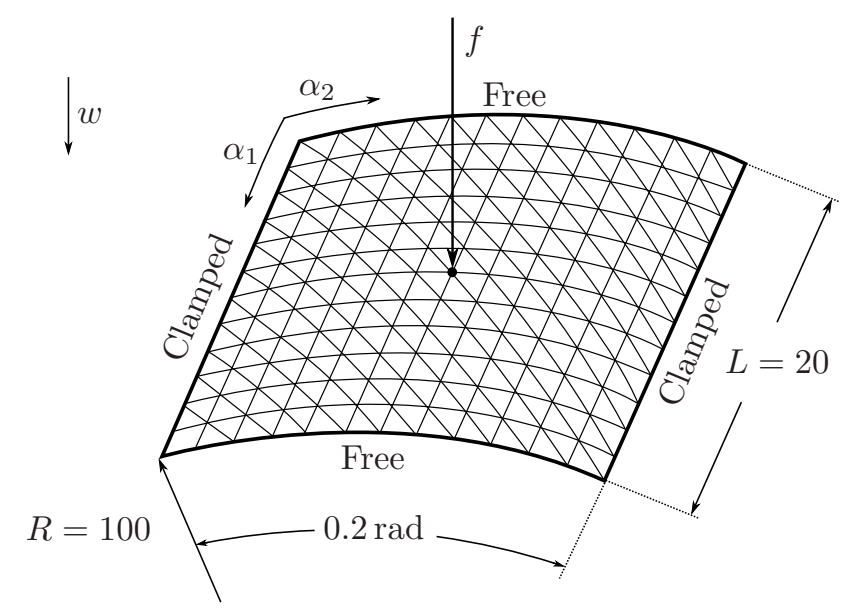

Figure 4: Clamped angle-ply cylindrical composite panel geometry

Table 3: Material properties - cylindrical composite panel

\begin{tabular}{cccccc}
\hline$E_{1}$ & $E_{2}$ & $\nu_{12}$ & $G_{12}$ & $G_{13}$ & $G_{23}$ \\
\hline $4.785 \cdot 10^{6}$ & $1.595 \cdot 10^{6}$ & 0.25 & $0.64 \cdot 10^{6}$ & $0.957 \cdot 10^{6}$ & $0.957 \cdot 10^{6}$ \\
\hline
\end{tabular}

are obtained using a full biquadratic (9-node) quadrilateral degenerate shell element. On the other hand, Kulikov and Plotnikova [42] used a linear (4-node) quadrilateral element based on a mixed formulation (Hu-Washizu variational principle). Fig. [5 gives the load-displacement curves for the center point for all three angle-ply laminates computed using the present element and the results reported by Kulikov and Plotnikova 42]. It also includes the results by Yeom and Lee 41] for the angle-ply [45/-45]. The results show very high agreement. 


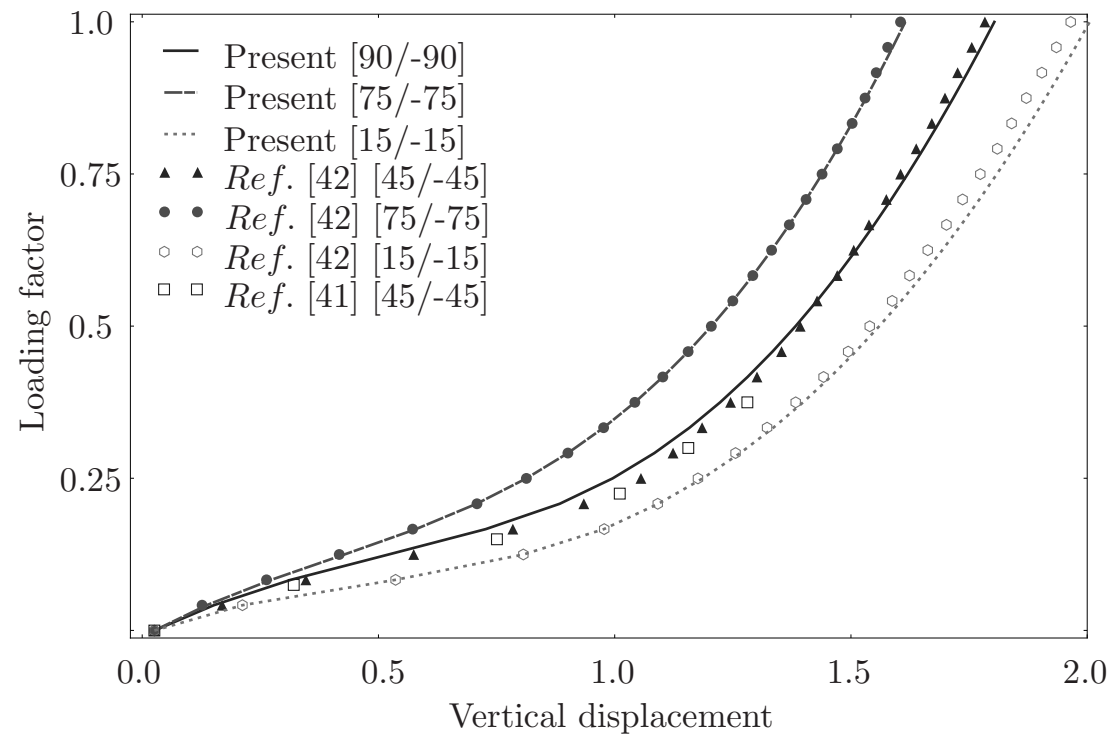

Figure 5: Load-displacment curves of the clamped angle-ply cylindrical panel case

\subsection{Snap-through of a clamped cylindrical shell}

145 example into extreme geometric nonlinearities. The considered structure is of a rather similar geometry. It is a cylindrical composite shell with a width of 1 shown together with its remaining dimensions in Fig. 6. The kinematic boundary conditions are exactly the same as in the previous case and the concentrated transverse force of $f=10000$ acts also at its center point. The shell is made of two layers, [0/90], with the $0^{\circ}$ - direction along the circumference. Both layers have the same thickness of 1 . The material properties are presented in Table 4 Similarly to the first considered case, due to the symmetry only a quater of the structure is modeled using 40 elements. The results reported by Li et al. [43]

Table 4: Material properties - laminated arch

\begin{tabular}{cccccc}
\hline$E_{1}$ & $E_{2}$ & $\nu_{12}$ & $G_{12}$ & $G_{13}$ & $G_{23}$ \\
\hline $25 \cdot 10^{6}$ & $1 \cdot 10^{6}$ & 0.3 & $0.2 \cdot 10^{6}$ & $0.5 \cdot 10^{6}$ & $0.5 \cdot 10^{6}$ \\
\hline
\end{tabular}




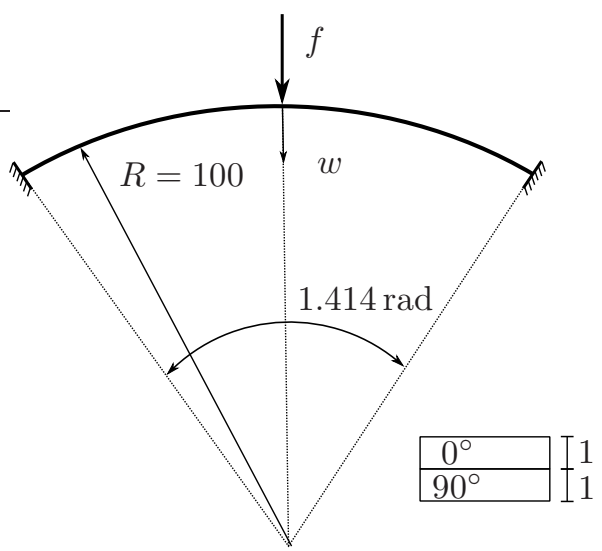

Figure 6: Clamped laminated arch geometry

155

obtained using a 4-node co-rotational quadrilateral composite shell element with the assumed natural strain approach. The load-displacement curves for the central point obtained by the present formulation and those reported by Li et al. [43] are illustrated in Fig. 7] Obviously, the problem at hand is characterized by the snap-through effect. It can be noticed that two results are reported by yields the results only up to the limit point, at which the solution becomes instable and the Newton-Raphson procedure fails to converge from that point. Upon switching to the static displacement-controlled computation (displacement increments), the complete curve is obtained. However, another solution procedure 165 is to get the static solution by computing a dynamic case with a relatively large damping, so that the structure comes to rest relatively quickly. This yields the result characterized by the almost horizontal line from the limit point to the part of the load-displacement curve at which the external force is incrementally 170 larger than at the limit point (Fig. 7). The diagram also shows that the results computed by the present element are practically congruent with those reported by Li et al. [43]. 


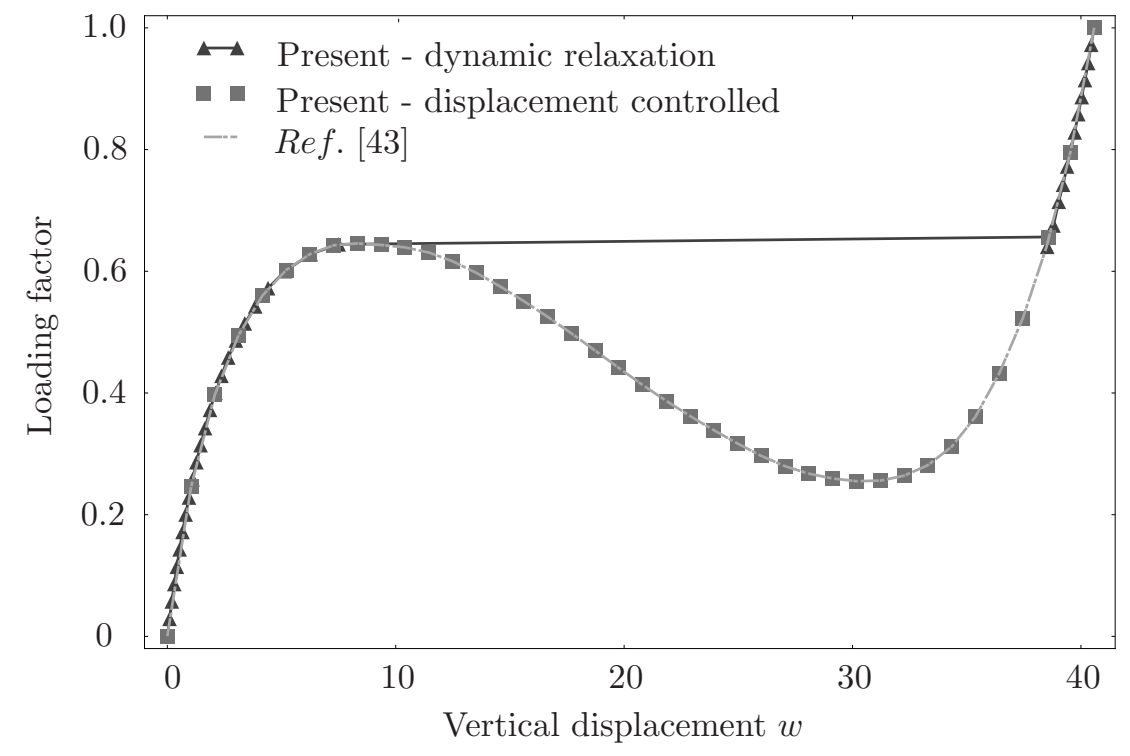

Figure 7: Load-displacement curves at mid-span of the laminated shallow arch

\subsection{Composite slit annular plate}

This example serves as one of the standard benchmark cases to test the developed finite elements in geometrically nonlinear analysis. The initial geometry is relatively simple (see Fig 8 ). It is a ring plate with a slit along the global $x$-direction across the whole width. One edge of the slit is exposed to a constant transverse line load $q=25$, while the other edge is clamped. The initial geometry with dimensions and the FE mesh involving 350 elements is depicted in Fig. 8, left. Choosing the material reference direction to run in the circumferential direction of the ring, two different composite stacking sequences are considered: $[0 / 0 / 0 /]$ and $[90 / 0 / 90]$, with the material properties as given in Table 5. Each layer has a thickness of 0.015. The diagram in Fig. 9] shows the

Table 5: Material properties - composite slit annular plate

\begin{tabular}{cccccc}
\hline$E_{1}$ & $E_{2}$ & $\nu_{12}$ & $G_{12}$ & $G_{13}$ & $G_{23}$ \\
\hline $2 \cdot 10^{6}$ & $0.6 \cdot 10^{6}$ & 0.3 & $0.24 \cdot 10^{6}$ & $0.3 \cdot 10^{6}$ & $0.4 \cdot 10^{6}$ \\
\hline
\end{tabular}

vertical displacements of points A and B against the load and for both stacking 


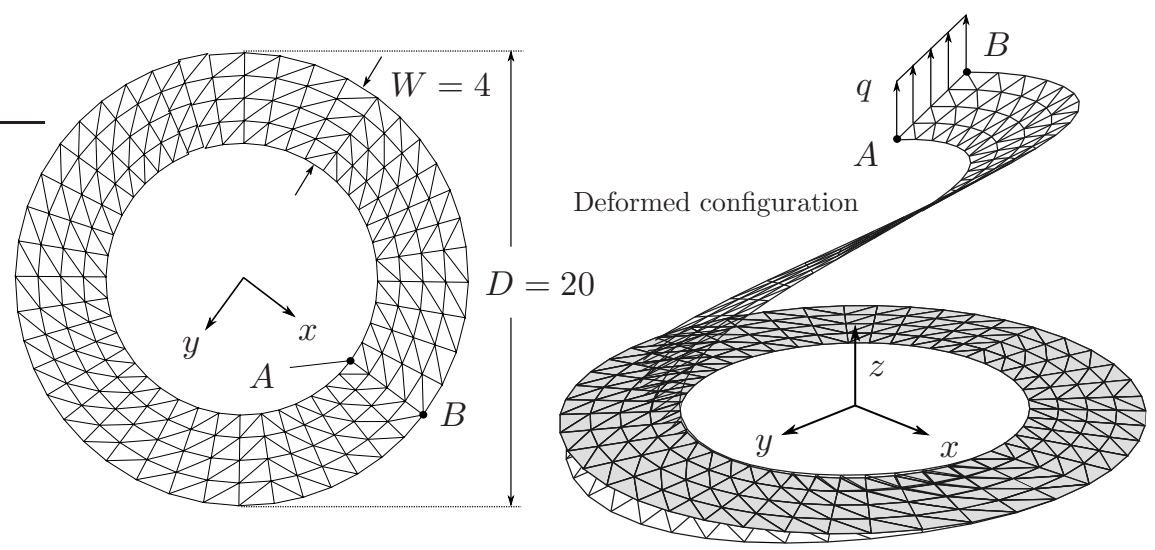

Figure 8: Composite slit annular plate

sequences. The obtained results are compared with those reported by Liang 44], who used a co-rotational quadratic triangular shell element with zig-zag functions. A good agreement between the results is observed.

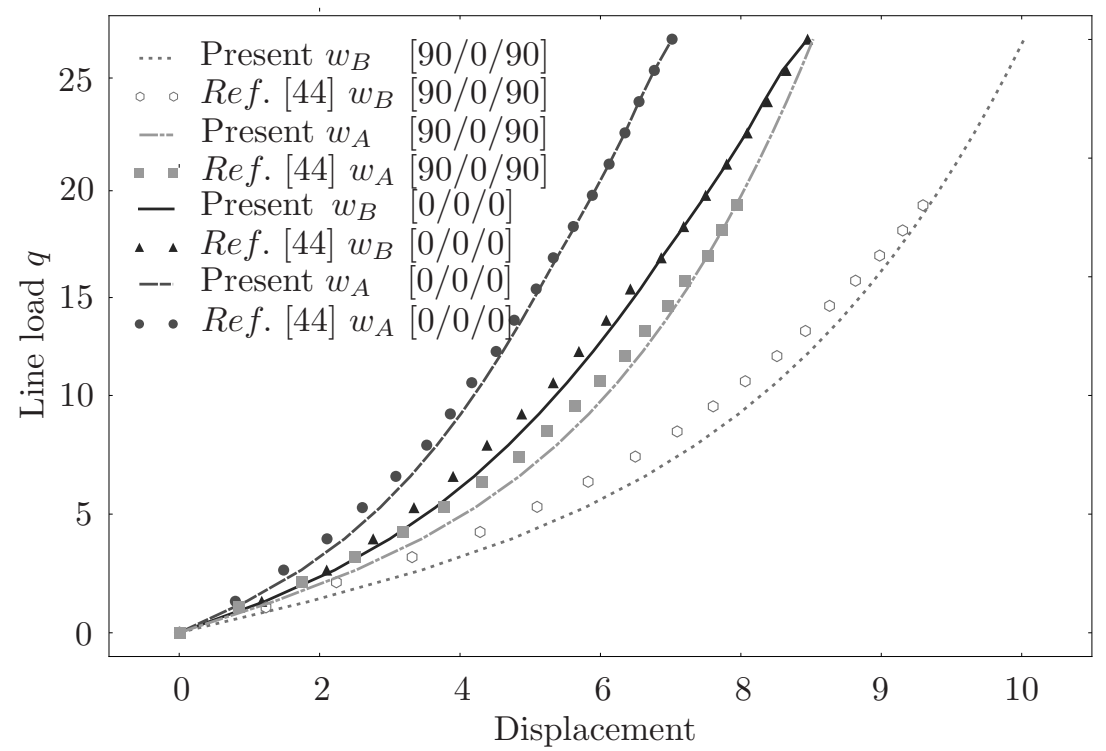

Figure 9: Load-displacment curves of the slit annular plate case 


\subsection{Modal analysis of a concrete boom pumps arm web segment}

The concrete boom pumps arm web segment depicted in Fig. 10] is made of FRC composite laminate consisting of 70 layers. A detailed description of the structural geometry and material data would take significant space. For the sake of brevity, it is omitted here. Another reason for this is the fact that the example does not belong to typical numerical benchmark cases that impose the need for repeatability. The aim is rather to compare numerical and experimental results in the case of a structure typically encountered in practice. The experimental
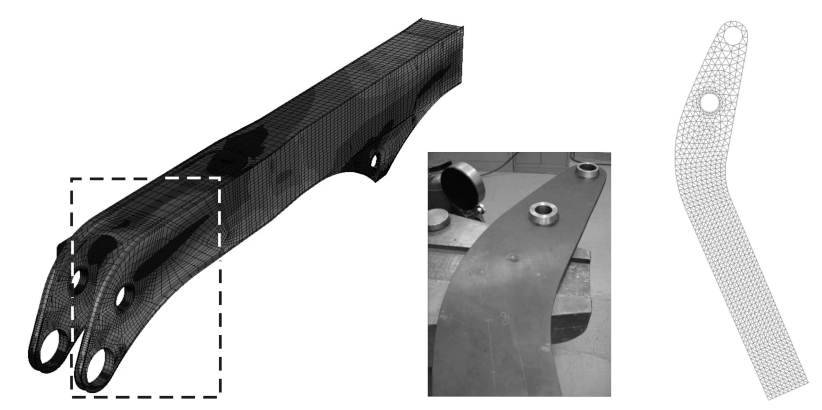

Figure 10: Arm web segment of a concrete boom pump

modal analysis of the investigated composite web structure was done by means of a laser scanning vibrometer (Polytech). The scanning-software defines its own mesh of measurement points over the real structure. For processing and comparing the measured and numerical modal data, the measurement points should be ideally coincident with the nodes of the FE model. This is not always easy to achieve in practice. Fig. 11 shows in parallel the real test object with the generated scanning mesh (the left-hand side) and the FE model, with the much finer FE mesh (the right-hand side). The marked nodes of the FE mesh are those that closely match the measurement points. The measured modal data and those from the FE analysis are compared based on the Modal Assurance Criteria (MAC) values and determined eigenfrequencies. The MAC value for the $i^{\text {th }}$ numerical mode, $\left\{\phi_{c i}\right\}$, and the $j^{\text {th }}$ experimentally determined mode, 


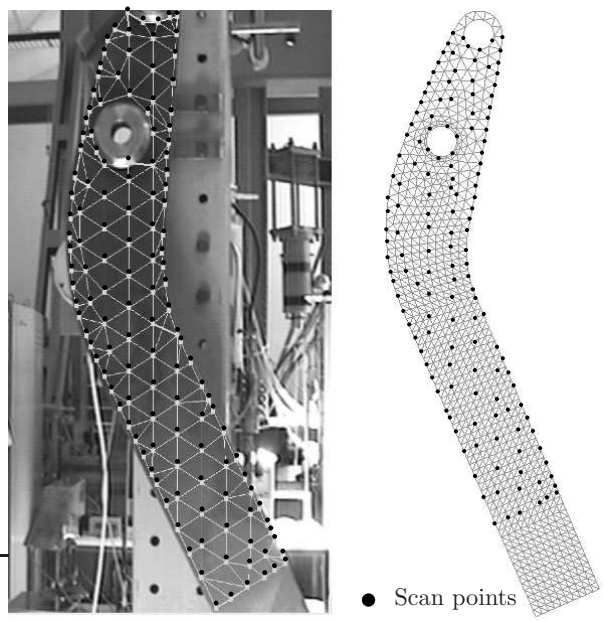

Figure 11: CFR composite web arm: real structure with the scanning mesh and the FE model with the nodes matching the scanning points

$\left\{\phi_{e j}\right\}$, is determined as:

$$
\text { MAC }=\frac{\left(\left\{\phi_{c i}\right\}^{T}\left\{\phi_{e j}\right\}\right)^{2}}{\left(\left\{\phi_{c i}\right\}^{T}\left\{\phi_{c i}\right\}\right)\left(\left\{\phi_{e j}\right\}^{T}\left\{\phi_{e j}\right\}\right)}
$$

The results are summarized in Table 6 for the first several modes. In the experiment, the structure was excited by a shaker acting perpendicularly to the web segment at one point (rather small surface). This excitation cannot excite the inplane modes nor the out-of-plane modes whose nodal lines run through or quite close to the position at which the shaker acts. For that reason no corresponding experimental modes were found for the $3^{\text {rd }}$ and $5^{\text {th }}$ numerically determined modes. Furthermore, though the MAC values in Table 6 show a clear correspondence between the remaining experimentally and numerically determined modes, some differences are still notable, particularly in higher modes. In fact, this was expectable, as the FE model represents the idealized version of the real structure. The considered real structure was laminated by hand, so that the thickness varies somewhat across the surface. Additionally, the clamped edge on the upper bushing does not represent an ideal clamp, as considered in the numerical simulation. The numerical model neglects the damping present in the real model. And finally, the comparison is done using the points that do 
not coincide, but are closely matched, which has a greater influence in more complex mode shapes.

Table 6: Modal Assurance Criterion (MAC) results

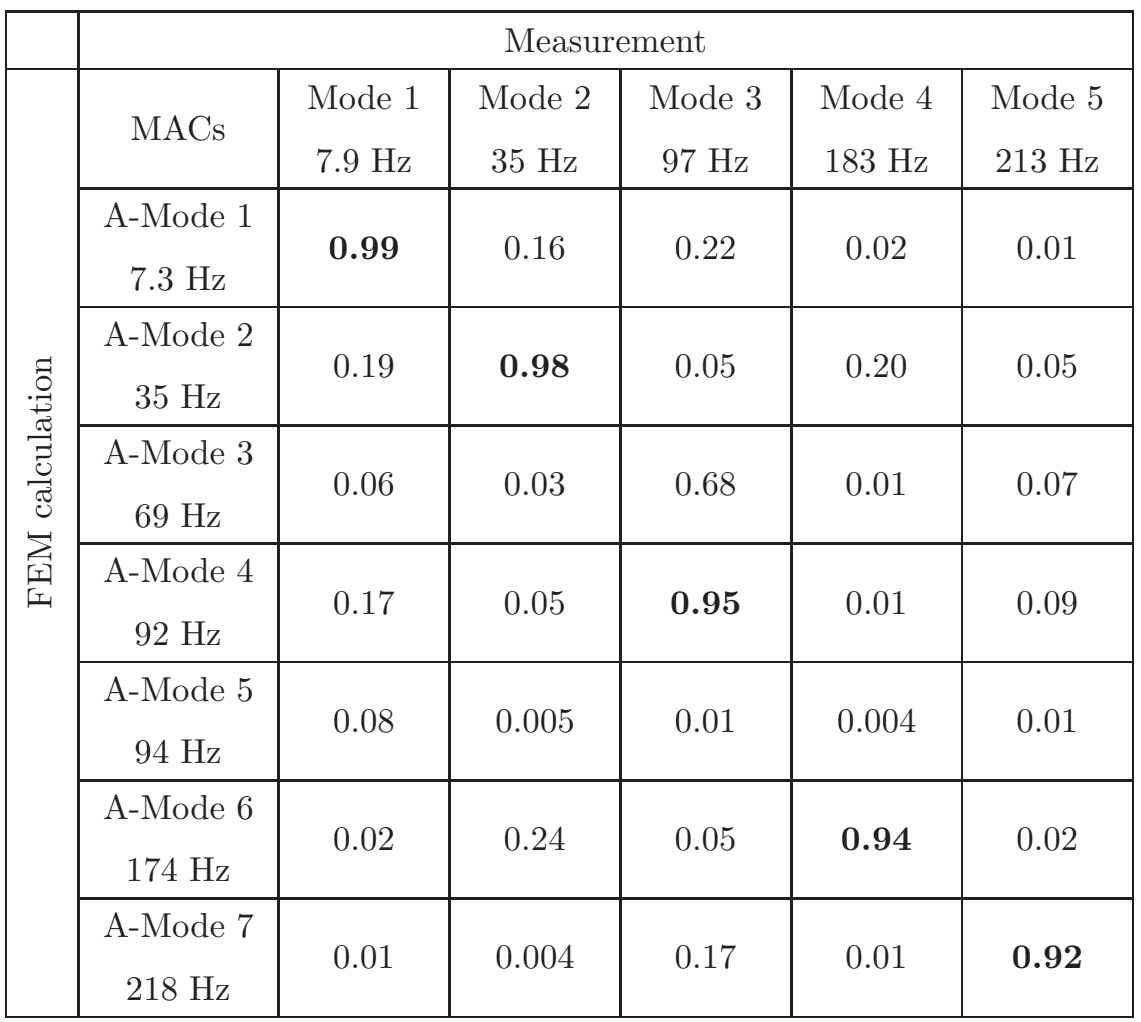

\section{Conclusion}

Thin-walled structures gain in importance and their field of application becomes more diverse each day. The improvement of their general properties and further growth are driven by application of modern engineering materials. The material architecture in form of FRC laminates opened up promising possibilities in design of thin-walled structures. At the same time, the complexity in the mechanical behavior of thin-walled structures became more emphasized thus calling for numerically efficient, accurate and reliable modeling tools.

The focus of the paper is on the proposed linear triangular shell element. 

indisputable, the general performance of linear shell elements, particularly triangular, has been the upsetting factor for the researchers and gave them therewith the impetus to improve the existing solutions. The developed shell element relies on the equivalent single-layer approach based on the Reissner-Mindlin implements already existing solutions for plate and membrane part, but as a new combination. The shear locking is resolved by means of the descrete shear gap method and the oscillations of the transverse shear stresses between elements are alleviated by means of the cell-smoothing technique. For the membrane behavThe element employs six degrees of freedom per node, whereby the drilling degree of freedom is of particular importance for the ANDES formulation of the membrane part. As thin-walled structures are susceptible to geometrically nonlinear behavior, the consistent co-rotational approach is implemented with the 230

The examples prove a very good performance of the element in both linear and geometrically nonlinear analyses. The examples cover both academic and practical cases. The validation was done using the results reported by other authors, those obtained by commercial FE codes and the experimental results were also addressed. Future work should extend the element formulation so as to include multi-functional materials and associated coupled-field effects.

\section{References}

[1] G. Li, F. Xu, G. Sun, Q. Li, Crashworthiness study on functionally graded thin-walled structures, International journal of crashworthiness 20 (2015) $280-300$.

[2] A. A. Parand, A. Alibeigloo, Static and vibration analysis of sandwich cylindrical shell with functionally graded core and viscoelastic interface using dqm, Composites Part B: Engineering 126 (2017) 1-16. 
[3] G. Kulikov, S. Plotnikova, E. Carrera, A robust, four-node, quadrilateral

[8] G. Rama, D. Marinkovi, M. Zehn, Efficient three-node finite shell element for linear and geometrically nonlinear analyses of piezoelectric laminated structures, Journal of Intelligent Material Systems and Structures 29 (2018) $345-357$.

[9] K. Rohwer, Models for intralaminar damage and failure of fiber compositesa review, Facta Universitatis-Series Mechanical Engineering 14 (2016) 119.

[10] N. Montinaro, D. Cerniglia, G. Pitarresi, Detection and characterisation of disbonds on fibre metal laminate hybrid composites by flying laser spot thermography, Composites Part B: Engineering 108 (2017) 164-173. 
[18] S. Valvano, E. Carrera, Multilayered plate elements with node-dependent kinematics for the analysis of composite and sandwich structures, Facta Universitatis-Series Mechanical Engineering 15 (2017) 1-30.

[19] P. Milić, D. Marinković, Isogeometric fe analysis of complex thin-walled

[11] T. Rademacher, M. Zehn, Modal triggered nonlinearities for damage localization in thin walled frc structures-a numerical study, Facta UniversitatisSeries Mechanical Engineering 14 (2016) 21-36.

[12] D. Marinković, H. Köppe, U. Gabbert, Degenerated shell element for geometrically nonlinear analysis of thin-walled piezoelectric active structures, Smart Materials and Structures 17 (2008) 015030.

[13] S. Jayasankar, S. Mahesh, S. Narayanan, C. Padmanabhan, Dynamic analysis of layered composite shells using nine node degenerate shell elements, Journal of Sound and Vibration 299 (2007) 1-11.

[14] Y. Zhang, C. Yang, Recent developments in finite element analysis for laminated composite plates, Composite Structures 88 (2009) 147-157.

[15] A. Ferreira, E. Carrera, M. Cinefra, C. Roque, Analysis of laminated doubly-curved shells by a layerwise theory and radial basis functions collocation, accounting for through-the-thickness deformations, Computational Mechanics 48 (2011) 13-25.

[16] R. Moreira, J. D. Rodrigues, A. Ferreira, A generalized layerwise finite element for multi-layer damping treatments, Computational Mechanics 37 (2006) 426.

[17] E. Carrera, A. Pagani, S. Valvano, Multilayered plate elements accounting for refined theories and node-dependent kinematics, Composites Part B: Engineering 114 (2017) 189-210.

structures, Transactions of FAMENA 39 (2015) 15-26. 
[20] C. H. Thai, H. Nguyen-Xuan, N. Nguyen-Thanh, T.-H. Le, T. NguyenThoi, T. Rabczuk, Static, free vibration, and buckling analysis of laminated composite reissner-mindlin plates using nurbs-based isogeometric approach, International Journal for Numerical Methods in Engineering 91 (2012) 571-603.

[21] S. Yin, T. Yu, T. Q. Bui, X. Zheng, S. Tanaka, In-plane material inhomogeneity of functionally graded plates: A higher-order shear deformation plate isogeometric analysis, Composites Part B: Engineering 106 (2016) 273-284.

[22] C. H. Thai, A. Ferreira, E. Carrera, H. Nguyen-Xuan, Isogeometric analysis of laminated composite and sandwich plates using a layerwise deformation theory, Composite Structures 104 (2013) 196-214.

[23] D. Marinković, G. Rama, Co-rotational shell element for numerical analysis of laminated piezoelectric composite structures, Composites Part B: Engineering 125 (2017) 144-156.

[24] K.-U. Bletzinger, M. Bischoff, E. Ramm, A unified approach for shearlocking-free triangular and rectangular shell finite elements, Computers \& Structures 75 (2000) 321-334.

[25] G.-R. Liu, N. T. Trung, Smoothed finite element methods, CRC press, 2016.

[26] J.-M. Berthelot, Composite materials: mechanical behavior and structural analysis, Springer Science \& Business Media, 2012.

[27] R. Rolfes, K. Rohwer, Improved transverse shear stresses in composite finite elements based on first order shear deformation theory, International Journal for Numerical Methods in Engineering 40 (1997) 51-60.

[28] C. A. Felippa, C. Militello, Membrane triangles with corner drilling freedomsii. the andes element, Finite Elements in Analysis and Design 12 (1992) 189-201. 
[29] P. Bergan, M. Nygård, Finite elements with increased freedom in choosing shape functions, International Journal for Numerical Methods in Engineering 20 (1984) 643-663.

[30] C. A. Felippa, A study of optimal membrane triangles with drilling freedoms, Computer Methods in Applied Mechanics and Engineering 192 (2003) 2125-2168.

[31] C. A. Felippa, S. Alexander, Membrane triangles with corner drilling freedoms-iii. implementation and performance evaluation, Finite Elements in Analysis and Design 12 (1992) 203-239.

[32] C. M. Shin, B. C. Lee, Development of a strain-smoothed three-node triangular flat shell element with drilling degrees of freedom, Finite Elements in Analysis and Design 86 (2014) 71-80.

[33] C. A. Felippa, B. Haugen, A unified formulation of small-strain corotational finite elements: I. theory, Computer Methods in Applied Mechanics and Engineering 194 (2005) 2285-2335.

[34] C. Rankin, F. Brogan, An element independent corotational procedure for the treatment of large rotations, Journal of pressure vessel technology 108 (1986) $165-174$.

[35] D. Marinkovic, M. Zehn, Z. Marinkovic, Finite element formulations for effective computations of geometrically nonlinear deformations, Advances in Engineering Software 50 (2012) 3-11.

[36] V. A. Nguyen, M. Zehn, D. Marinković, An efficient co-rotational fem formulation using a projector matrix, Facta Universitatis-Series Mechanical Engineering 14 (2016) 227-240.

[37] B. Nour-Omid, C. Rankin, Finite rotation analysis and consistent linearization using projectors, Computer Methods in Applied Mechanics and Engineering 93 (1991) 353-384. 
[38] J. Argyris, An excursion into large rotations, Computer methods in applied mechanics and engineering 32 (1982) 85-155.

[39] National Agency for Finite Element Methods and Standards - NAFEMS, (accessed April 12, 2018). Https://www.nafems.org.

[40] Composites Benchmarks Test R0031/1, 1995. NAFEMS publication R0031.

[41] C. Yeom, S. Lee, An assumed strain finite element model for large deflection composite shells, International Journal for Numerical Methods in Engineering 28 (1989) 1749-1768.

[42] G. Kulikov, S. Plotnikova, Non-linear strain-displacement equations exactly representing large rigid-body motions. part i timoshenko-mindlin shell theory, Computer Methods in Applied Mechanics and Engineering 192 (2003) 851-875.

[43] Z. Li, T. Zheng, L. Vu-Quoc, B. Izzuddin, A 4-node co-rotational quadrilateral composite shell element, International Journal of Structural Stability and Dynamics 16 (2016) 1550053.

[44] Y. Liang, Nonlinear analysis of composite shells with application to glass structures, dissertation, Imperial College London, 2015. 